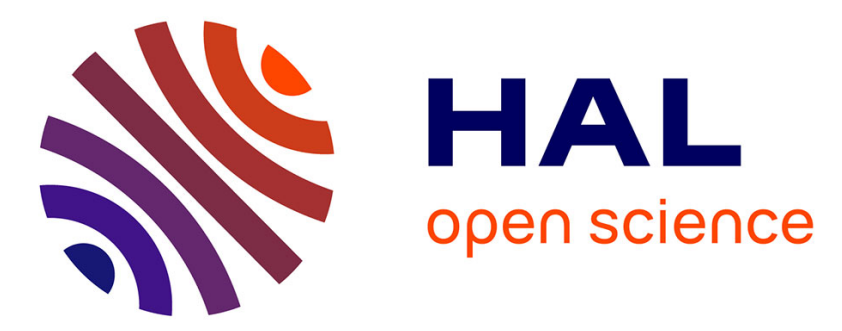

\title{
Instationary Eulerian viscoelastic flow simulations using time separable Rivlin-Sawyers constitutive equations
}

\author{
E.A.J.F. Peters, M.A. Hulsen, B.H.A.A. van den Brule
}

\section{To cite this version:}

E.A.J.F. Peters, M.A. Hulsen, B.H.A.A. van den Brule. Instationary Eulerian viscoelastic flow simulations using time separable Rivlin-Sawyers constitutive equations. Journal of Non-Newtonian Fluid Mechanics, 2000, 89 (1-2), pp.209 - 228. 10.1016/S0377-0257(99)00026-9 . hal-01722610

\section{HAL Id: hal-01722610 https://hal.science/hal-01722610}

Submitted on 4 Mar 2018

HAL is a multi-disciplinary open access archive for the deposit and dissemination of scientific research documents, whether they are published or not. The documents may come from teaching and research institutions in France or abroad, or from public or private research centers.
L'archive ouverte pluridisciplinaire HAL, est destinée au dépôt et à la diffusion de documents scientifiques de niveau recherche, publiés ou non, émanant des établissements d'enseignement et de recherche français ou étrangers, des laboratoires publics ou privés. 


\title{
Instationary Eulerian viscoelastic flow simulations using time separable Rivlin-Sawyers constitutive equations
}

\author{
E.A.J.F. Peters, M.A. Hulsen, B.H.A.A. van den Brule \\ Delft University of Technology, J.M. Burgers Centre for Fluid Mechanics, Rotterdamseweg 145, \\ 2628AL Delft, The Netherlands
}

\begin{abstract}
A time dependent method for solving integral constitutive equations of the Rivlin-Sawyers type is introduced. The deformation history is represented by a finite number of deformation fields. Using these fields the stress integral is approximated as a finite sum. When the flow evolves the deformation fields are convected and deformed. The approach presented in this paper is the first Eulerian method that can handle integral equations in a time dependent way. The method is validated by using the upper-convected Maxwell (UCM) benchmark of a sphere moving in a tube. We show that the method converges with mesh and time step refinement and that the results are accurate, comparable to the results obtained with the differential equivalent of the UCM model. To demonstrate that complicated linear spectra are easily incorporated, results of a Rouse model simulation of 100 modes are presented. We also compare results on a falling sphere problem to the results obtained by a Lagrangian method as reported by Rasmussen and Hassager [H.K. Rasmussen, O. Hassager, On the sedimentation velocity of spheres in a polymeric liquid, Chem. Eng. Sci. 51 (1996) 1431-1440]. The model being employed is the PSM model, for which no differential equivalent exists.
\end{abstract}

Keywords: Integral models; Eulerian description; Falling sphere in tube; Transient viscoelastic flow; DEVSS/DG

\section{Introduction}

In the analysis of a viscoelastic fluid flowing through a complex domain one usually has to resort to a numerical simulation of the problem. In an isothermal problem the balance equations for momentum and mass have to be solved together with a constitutive model that expresses the stress in the material in terms of the deformation history. In most numerical studies the constitutive model is of a differential type. Differential constitutive equations have the numerical advantage that the evolution of the stress at a certain instant in time depends on the current velocity and stress fields only. Using a differential constitutive equation has, therefore, the clear advantage that it is not necessary to memorise the 
complete history of the flow problem. On the other hand, many (potentially) successful constitutive models are not of a differential type. These more promising constitutive equations include the kinetic micro-rheological models and also integral models. Some of the more successful constitutive models to describe the behaviour of a polymer melt, such as the equations following from reptation theory, are of the integral type. In recent years there is a trend to incorporate these sophisticated models directly into the simulation of the flow of a macro-molecular fluid without resorting to an approximation by a differential type model.

To incorporate a kinetic model directly into a flow simulation, thus by-passing the need of a closed form constitutive equation, the 'CONNFFESSIT' approach was developed by Laso and Öttinger [2]. Their original method was a particle tracking method with uncorrelated stochastic processes acting on each particle. An Eulerian approach to incorporate Brownian dynamics simulations in a viscoelastic flow simulation was developed by Hulsen, van Heel and van den Brule [3]. In this method the molecular orientations are stored in an ensemble of configuration fields. The stochastic process is taken to be the same everywhere within a configuration field while the noise in different fields remains uncorrelated. This completely removes the noise in spatial derivatives and ensures the continuity of the fields. In addition to the strong reduction of the noise in the simulation, the configuration field method offers the additional advantage that the size of the molecular ensemble is independent of the local size of the mesh, thus avoiding the problems associated with mesh refinement in a Lagrangian implementation. Recently Gallez et al. [4] presented an improved variant of the Lagrangian particle CONNFFESSIT approach where they introduced correlation between sub-ensembles of molecules in different fluid particles, similar to configuration fields. Furthermore, a method to continuously create and destruct particles is introduced, which makes the Lagrangian particle method also suitable for a problem with a highly graded mesh.

To evaluate a flow problem using an integral constitutive equation, it seems most natural at first to use a Lagrangian description of the flow field; the memory integral can be relatively easily evaluated following the fluid particle. The main drawback of this approach is that in a Lagrangian description the computational mesh deforms with the fluid. In a strong flow field this means that regular remeshing is required to maintain numerical accuracy. This is not only cumbersome but also leads to a loss of accuracy since in the remeshing procedure one has to interpolate the solution on the old mesh in order to find the values of the relevant variables at the nodal points of the new mesh. Another less attractive element of a Lagrangian implementation is that in order to achieve a refinement of the mesh somewhere in a problematic area downstream in the flow field one already has to know upstream how to construct the mesh. A good example of a Lagrangian approach to viscoelastic flow modelling can be found in the papers by Rasmussen and Hassager. In the first two papers [5,6] they used an upper convected Maxwell model (UCM) to model the flow past a sphere falling in a cylindrical tube. Because the UCM model is of the differential type it is, in principle, not necessary to memorise the whole flow history. In a later paper [1] they used the Papanastasiou-Scriven-Macosko model (PSM) which is a member of the Rivlin-Sawyers class of equations. This equation does not have a differential analogue. In the simulation all the previous coordinates of the deforming mesh have to be stored in order to be able to reconstruct the deformation history.

In an Eulerian approach all the problems associated with the deformation of the mesh are of course absent. The most common strategy to use an integral equation in this setting is to evaluate the stress at a certain point in the flow domain integrating backwards along the particle track (see e.g. [7,8]). The drawback of this method is that such a procedure is only feasible for steady flows. For time-dependent 
flows the complete particle tracks have to reconstructed again at every time step. To our knowledge nobody has ever tried a time dependent particle (back)tracking method to solve integral constitutive equations.

In this paper, we will introduce a time dependent Eulerian technique for implementing integral constitutive equations, bypassing the problems associated with mesh deformation without being limited to steady-state problems. In the next section the basic concept of the deformation field method will be explained. Then, in Sections 3 and 4 we will present the relevant details of the numerical implementation. In Section 5 the results obtained on the falling sphere benchmark problem will be compared against data reported by other groups. We will first compare our results obtained for an integral representation of the UCM model with the extensive data that can be found in the literature. Then we will compare simulations with the PSM model against the results obtained by Rasmussen and Hassager.

\section{The basic concept of the deformation field approach}

In this paper, we will consider the isothermal flow of a viscoelastic fluid in a complex domain. It is assumed that inertial effects are negligible and that the fluid is incompressible. Under these assumptions the flow is governed by the momentum balance which reduces to

$$
-\nabla \cdot \boldsymbol{\sigma}(\boldsymbol{x}, t)=-\eta_{s} \nabla^{2} \boldsymbol{v}(\boldsymbol{x}, t)-\nabla \cdot \tau(\boldsymbol{x}, t)+\nabla p(\boldsymbol{x}, t)=0,
$$

and the mass balance which reduces to

$$
\nabla \cdot \boldsymbol{v}(\boldsymbol{x}, t)=0
$$

where $\boldsymbol{v}(\boldsymbol{x}, t)$ is the velocity field. The total stress $\boldsymbol{\sigma}$ has three contributions: a viscous contribution $\eta_{\mathrm{s}}\left(\nabla \boldsymbol{v}+\nabla \boldsymbol{v}^{\mathrm{T}}\right)$, a viscoelastic extra-stress $\tau$ and an isotropic pressure contribution $-p \mathbf{1}$. The viscous contribution may be absent for some problems. In order to close the set of equations, a constitutive relation is needed which expresses the extra stress $\tau$ in terms of the history of deformation. In this paper we will use a Rivlin-Sawyers integral equation.

For the time separable Rivlin-Sawyers model the stress tensor is given by the following relation

$$
\tau(\boldsymbol{x}, t)=\int_{-\infty}^{t} M\left(t-t^{\prime}\right) f\left[\boldsymbol{B}_{t^{\prime}}(\boldsymbol{x}, t)\right] \mathrm{d} t^{\prime},
$$

where $M(t)$ is the memory function, $f$ is an isotropic function and $\boldsymbol{B}_{t^{\prime}}(\boldsymbol{x}, t)$ is the Finger strain tensor. $\boldsymbol{B}_{t^{\prime}}(\boldsymbol{x}, t)$ is a field that measures the deformation of a fluid element currently present at position $\boldsymbol{x}$, with respect to the reference time $t^{\prime}$ somewhere in the past; so by definition, at the moment of 'creation' $\boldsymbol{B}_{t^{\prime}}\left(\boldsymbol{x}, t^{\prime}\right)=\mathbf{1}$, where $\mathbf{1}$ is the unit tensor. The time evolution of the Finger tensor is governed by

$$
\stackrel{\nabla}{\boldsymbol{B}_{t^{\prime}}}=0
$$

or

$$
\frac{\partial}{\partial t} \boldsymbol{B}_{t^{\prime}}(\boldsymbol{x}, t)+\boldsymbol{v}(\boldsymbol{x}, t) \cdot \nabla \boldsymbol{B}_{t^{\prime}}(x, t)=\nabla \boldsymbol{v}^{\mathrm{T}}(\boldsymbol{x}, t) \cdot \boldsymbol{B}_{t^{\prime}}(\boldsymbol{x}, t)+\boldsymbol{B}_{t^{\prime}}(\boldsymbol{x}, t) \cdot \nabla \boldsymbol{v}(\boldsymbol{x}, t),
$$


where one should keep in mind that $t^{\prime}$ is a reference time that should be kept fixed. So, one way to read the Rivlin-Sawyers equation is that at every instant in time a Finger tensor field is created (as a unit tensor). This field is labelled by the time $t^{\prime}$ of its creation. After creation, the field is deformed and convected by the flow, obeying Eq. (5). The second term on the left-hand side (LHS) of Eq. (5) accounts for the convective motion of the field. This convective term removes the need to track the motion of fluid particles. The terms on the RHS describe the further deformation of the field caused by the presence of a velocity gradient. The important thing to notice in Eq. (5), and the key of our approach, is that the evaluation of the time evolution of $\boldsymbol{B}_{t^{\prime}}(\boldsymbol{x}, t)$ only requires information of the velocity field at the current time $t$. All information about the flow history is contained in the Finger tensors $\boldsymbol{B}_{t^{\prime}}(\boldsymbol{x}, t)$ with $t^{\prime}<t$. In the actual implementation not the full tensors are stored and updated, but the number of components is reduced by one. This is accomplished by using the fact that $\operatorname{det}\left(\boldsymbol{B}_{t^{\prime}}\right)=1$. The details are given in Appendix A. Apart from reducing the storage and CPU requirements there also seems to be a slight improvement in accuracy, but further study is needed to give a more definite statement on this.

The contribution of a particular field to the stress at the current time $t$ is weighted by the memory function and depends on the age of the field, i.e. $t-t^{\prime}$. To construct the memory integral one would ideally like to know $f\left[\boldsymbol{B}_{t^{\prime}}(\boldsymbol{x}, t)\right]$ for all $t^{\prime}$. To approximate this infinite amount of information, $f\left[\boldsymbol{B}_{t^{\prime}}\right]$ will be discretised with respect to the reference time $t^{\prime}$. Furthermore, a cut-off time $\tau_{\mathrm{c}}$ is introduced by limiting the reference time to $t-\tau_{\mathrm{c}}<t^{\prime}<t$. To reconstruct the discretised version of $f\left[\boldsymbol{B}_{t^{\prime}}\right]$, the tensor fields $\boldsymbol{B}_{t^{\prime}}$ only have to be stored for a finite number of reference times $t^{\prime}$. In this way we obtain a manageable number of fields, the deformation fields, each labelled by their time of creation.

The functions $f\left[\boldsymbol{B}_{t^{\prime}}\right]$ for a continuous $t^{\prime}$ are approximated by means of an interpolation between the discrete fields created at times $t-\tau_{i}$ in the past. For this reason linear base functions $\phi_{i}(\tau)$ are introduced (see Fig. 1),

$$
f\left[\boldsymbol{B}_{t-\tau}(t)\right]=\sum_{i=0}^{N-1} f\left[\boldsymbol{B}_{t-\tau_{i}}(t)\right] \phi_{i}(\tau) .
$$

A special choice is made for the last base function. This is an especially good choice in startup problems. In startup problems (with startup at $t=0$ ) all $\boldsymbol{B}_{t^{\prime}}$ with $t^{\prime}<0$ are equal. The choice of taking the base function to be 1 for $\tau>\tau_{\mathrm{c}}$ makes that there is no cut-off error for times $t<\tau_{\mathrm{c}}$. Substitution of Eq. (6) into Eq. (3) yields

$$
\tau(\boldsymbol{x}, t)=\sum_{i} W_{i} f\left[\boldsymbol{B}_{t-\tau_{i}}(\boldsymbol{x}, t)\right]
$$

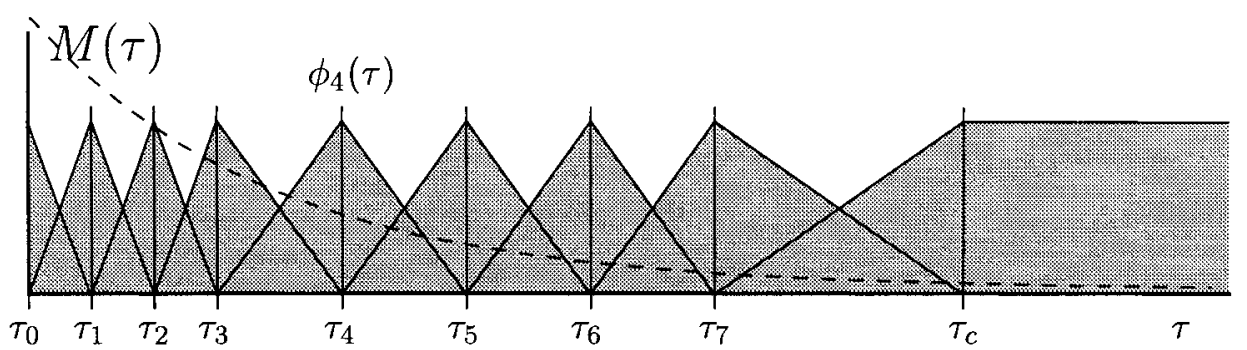

Fig. 1. The values tensors $f\left[\boldsymbol{B}_{t-\tau}(t)\right]$ are only known for discrete ages $\tau_{i}$. For intermediate ages we use the base function $\phi_{i}(\tau)$ to make an interpolation: $f\left[\boldsymbol{B}_{t-\tau}(t)\right]=\sum_{i} f\left[\boldsymbol{B}_{t-\tau_{i}}(t)\right] \phi_{i}(\tau)$. In this figure we depicted the base functions used in our case (linear interpolation). 
where the weights $W_{i}$ are given by

$$
W_{i}=\int_{0}^{\infty} M(\tau) \phi_{i}(\tau) \mathrm{d} \tau .
$$

The weights $W_{i}$ only have to be calculated once, before the start of the actual flow simulation. The stress integral is approximated by a finite sum and is second order in time. If $f\left[\boldsymbol{B}_{t^{\prime}}\right]$ is bounded and $\tau_{\mathrm{c}}$ is of the order of a few times the largest relaxation time, the error due to this truncation will be relatively small since most realistic memory functions decay exponentially for large times. There are models where $f$ is not bounded, e.g. the Maxwell or Rouse model. The stress in these models can diverge in a strong elongational flow. The errors due to truncation of the memory function can under these circumstances become very large. The possibility to grow without bound indicates a serious flaw in the constitutive equation. For the Rouse chain e.g. the underlying problem is the infinite extensibility at large but finite rates of elongation.

\section{The discretisation of the reference time}

Old fields will contribute very little to the stress since the memory function is a decreasing function of time. Therefore a cut-off time $\tau_{\mathrm{c}}$ is introduced, as explained in the previous paragraph. This also offers the possibility, at every time step, to annihilate an old field. In this way memory space is created to store the newly created field which carries the current time as a label. Using this procedure the number of fields is kept constant during the simulation.

To keep things clear, we will first present the simplest approach to the implementation of deformation fields. The time discretisation of the deformation fields will be taken to be equidistant and we will use the same time increment $\Delta t$ as the one used in the flow simulation to solve the balance equations. The update algorithm of the deformation fields consists of three steps:

- Updating of the existing fields, i.e. the convection and deformation of the memory fields.

$$
\boldsymbol{B}_{t-i \Delta t}(t) \rightarrow \boldsymbol{B}_{t-i \Delta t}(t+\Delta t)
$$

- Annihilation of an old field. In the simple approach, equidistant discretisation, the oldest field will be annihilated.

$$
\boldsymbol{B}_{t-\tau_{\mathrm{c}}}(t) \rightarrow \mathrm{NULL}
$$

- Creation of a new field. Using the memory space released by annihilation of an old field a new field is created as a unit tensor field with reference time $t+\Delta t$.

$$
\boldsymbol{B}_{t+\Delta t}(t+\Delta t):=\mathbf{1}
$$

In addition to the updating of the fields the weights associated to each field have to be decreased since they aged by a time increment. The field $\boldsymbol{B}_{t-i \Delta t}(t)$ was $i$ time steps old and therefore $f\left[\boldsymbol{B}_{t-i \Delta t}(t)\right]$ attributes with weight $W_{i}$ to stress tensor. The updated field is $i+1$ time steps old and has a new, and lower, weight $W_{i+1}$. This is illustrated in Fig. 2. Using the new weights, the stress throughout the flow domain can be calculated. With the new stresses the velocity field can be updated etc. 




Fig. 2. The infinite number of fields $\boldsymbol{B}_{t^{\prime}}(\boldsymbol{x}, t)$ is represented by a finite number of fields. This is done by introducing a cut-off time, and by discretising the integral.

Since the time step for the evaluation of the memory integral has to obey other criteria than the time step used for solving the momentum balance and the update of the fields, it is not very convenient to be forced to use the same time increment for both processes. With respect to the time discretisation of the memory function one can remark that the weight decreases with age and that usually the decrease of the memory function will be steeper for small times. For this reason one would like to have the required small time increments at a short time scale which enable us to resolve the fast modes of the fluid whereas at the longer time scales the accuracy can be less since the weight is low. Errors made here contribute relatively little to the total stress.

There are many ways to discretise the memory integral. In the current implementation the past time $t^{\prime} \leq t$ is divided into a number of intervals. Each interval is divided into a number of equidistant time steps. In the first interval (youngest fields) this step is equal to the time step used in the flow simulation. For older fields larger time steps are taken and usually found by doubling the value in the previous interval. An example of such a discretisation is given by the first column of Fig. 3.

All weights needed in a computation of the stress are calculated in advance. There is however some minor bookkeeping to assign the correct weight to each of the deformation fields. This bookkeeping has to be repeated at each time step (see Fig. 3). For the second-order accurate algorithm the weight associated to a field can be reconstructed when, in addition to its own age, the age of the preceding and the following fields are known. The base function corresponding to the field can then be determined and the weight is easily calculated using Eq. (8).

We found that about 100 deformation fields were sufficient to represent the deformation history with a high accuracy. The optimal distribution of the time intervals can differ slightly for different memory functions. 


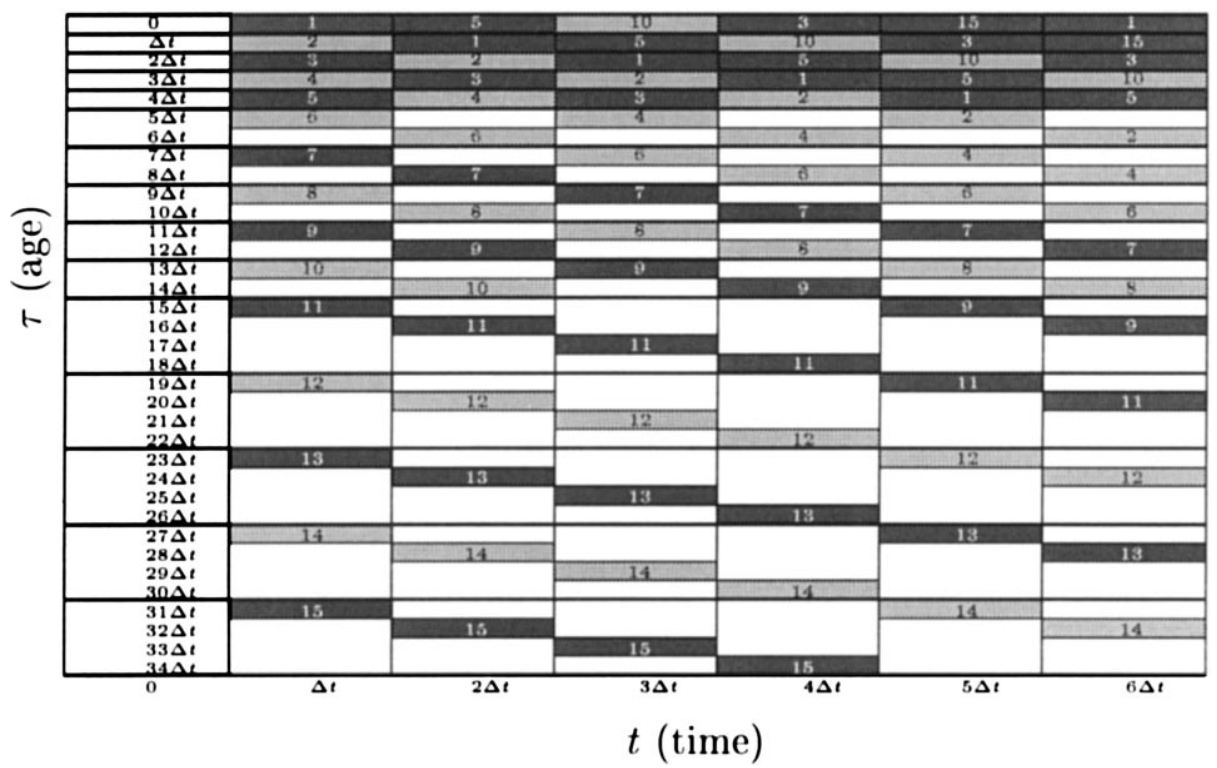

Fig. 3. Visualisation of the process of the aging of 15 deformation fields. Each box containing a number represents a field. The number refers to the part of the computer memory where the field is stored. At selected instances the time step is doubled. In this way the discretisation becomes fine for young fields. The discretisation is allowed to become coarser with increasing age of the fields. More fresh fields are created than old fields are needed. This means that fields are selectively annihilated at a certain age.

\section{Numerical methods}

In addition to the discretisation of the memory integral, as discussed in Section 3, we have to discretise several partial differential equations as well. The methods we use are similar to those used by Hulsen et al. [3] for solving stochastic models. Here we will briefly summarise the methods and refer to [3] for any details.

For the spatial discretisation we will use the finite element method. In particular we use the discrete elastic-viscous split stress (DEVSS) formulation of Guénette and Fortin [9] for the discretisation of the linear momentum balance and the continuity equation. This method leads to an additional variable $\boldsymbol{e}=\eta_{\mathrm{p}}\left(\nabla \boldsymbol{v}+\nabla \boldsymbol{v}^{\mathrm{T}}\right)$, the viscous polymer stress, where $\eta_{\mathrm{p}}$ is the contribution to the zero-shear-rate viscosity of the stress tensor $\tau$. The discontinuous Galerkin (DG) formulation will be used to discretise the evolution equation for the $\boldsymbol{B}_{t^{\prime}}$ fields given by Eq. (5). The discrete form of the equations is obtained by requiring that the weak form is valid on approximating subspaces which consist of piecewise polynomial spaces. In this work, we use quadrilateral elements with continuous biquadratic polynomials $\left(Q_{2}\right)$ for the velocity, discontinuous linear polynomials $\left(P_{1}\right)$ for the pressure, continuous bilinear polynomials $\left(Q_{1}\right)$ for the viscous polymer stress and discontinuous bilinear polynomials $\left(Q_{1}\right)$ for the deformation fields.

For the time discretisation of the evolution equation Eq. (5) we use an explicit Euler scheme. Therefore, the Finger tensor at the next time step $\boldsymbol{B}_{t^{\prime}}^{n+1}$ is computed from the velocity and the Finger tensor at the current time as follows

$$
\boldsymbol{B}_{t^{\prime}}^{n+1}=\boldsymbol{B}_{t^{\prime}}^{n}+\Delta t\left[-\boldsymbol{v}^{n} \cdot \nabla \boldsymbol{B}_{t^{\prime}}^{n}+\left(\nabla \boldsymbol{v}^{n}\right)^{\mathrm{T}} \cdot \boldsymbol{B}_{t^{\prime}}^{n}+\boldsymbol{B}_{t^{\prime}}^{n} \cdot \nabla \boldsymbol{v}^{n}\right]
$$


where we omitted the dependence on $\boldsymbol{x}$. The stress tensor $\tau^{n+1}$ can be found from Eq. (3) by using $\boldsymbol{B}_{t^{\prime}}^{n+1}$, just evaluated. The velocity and pressure field at the next time step, $v^{n+1}$ and $p^{n+1}$, are obtained from the momentum balance and the continuity equation:

$$
\begin{aligned}
& -\eta_{\mathrm{s}} \nabla^{2} \boldsymbol{v}^{n+1}+\nabla p^{n+1}=\nabla \cdot \tau^{n+1}, \\
& \nabla \cdot \boldsymbol{v}^{n+1}=0 .
\end{aligned}
$$

If the solvent viscosity $\eta_{\mathrm{s}}$ is zero or very small, the latter procedure is not possible and we have to proceed differently. In that case we add the term $E$ given by

$$
E=\bar{\eta} \nabla^{2}\left(\boldsymbol{v}^{n+1}-\boldsymbol{v}^{n}\right),
$$

to the momentum equation. This leads to an approximate momentum equation

$$
-\left(\eta_{\mathrm{s}}+\bar{\eta}\right) \nabla^{2} \boldsymbol{v}^{n+1}+\nabla p^{n+1}=-\bar{\eta} \nabla^{2} \boldsymbol{v}^{n}+\nabla \cdot \tau^{n+1}
$$

instead of Eq. (13). It is usually sufficient to take $\bar{\eta}=G \Delta t$, where $G$ is a typical shear modulus for the model. This means that $E$, and thus the error in the momentum equation, is of the order $\Delta t^{2}$. Note that $E=0$ if the flow becomes steady.

In the actual implementation we use a $2 \times 2$ Gauss integration scheme to integrate the weak form of Eq. (12) and a $3 \times 3$ scheme for Eqs. (13) and (14). The stress tensor $\tau$ is obtained by directly evaluating Eq. (3) in the $2 \times 2$ Gauss points. The value of $\tau$ in the $3 \times 3$ Gauss points, as needed in Eq. (13), is obtained by interpolating with a bilinear polynomial $\left(Q_{1}\right)$. This means that we actually use a projection of the stress tensor on the $Q_{1}$ space, instead of an evaluation of Eq. (3) in the $3 \times 3$ Gauss points. The difference is only relevant if the function $f$ in Eq. (3) is nonlinear.

\section{Validation of the method}

To demonstrate the use and the merits of the new approach three different constitutive models will be evaluated in a complex flow problem. To this end we selected the well-known geometry of a sphere moving along the centreline of a cylindrical tube.

The first set of data that will be shown concerns the simulation of a sphere moving through a fluid described by the upper-convected Maxwell model (UCM). Because the UCM model also has an integral formulation, the deformation field method can be used. The results will be compared with those obtained from various differential approaches. There is a large amount of high quality data available. This enables us to compare the accuracy of the deformation field method with other approaches.

The UCM model is very basic. It only has a single relaxation time and the memory integral has a linear dependence on the Finger tensor. To illustrate that the deformation field method can handle all types of Rivlin-Sawyers equations, and to show the many possibilities of the method, two variations on the UCM integral constitutive equation will be presented. Firstly, the time dependent part will be varied. This part, the memory function, fully determines the linear viscoelastic behaviour of the fluid. The results for a Rouse spectrum consisting of 100 modes will be presented. The second obvious modification is the dependency of the memory integral on the strain. This provides the possibility to modify the behaviour of the model in the non-linear regime such as e.g. the amount of shear thinning 
and the behaviour of the fluid in strong elongational flow. As an example the Papanastasiou-ScrivenMacosko (PSM) model will be simulated. The model parameters and the flow geometry selected in this simulation correspond to a problem simulated by Rasmussen and Hassager [1]. The results will be compared to the results obtained by these authors.

\subsection{The upper-convected Maxwell model}

The memory integral for the stress of the UCM model is

$$
\tau=\frac{\eta}{\lambda^{2}} \int_{-\infty}^{t} \exp \left[\frac{\left(t^{\prime}-t\right)}{\lambda}\right] \boldsymbol{B}_{t^{\prime}} \mathrm{d} t^{\prime}
$$

where $\lambda$ is the relaxation time of the fluid and $\eta$ is the viscosity. There is no solvent contribution to the stress.

The problem we consider here is a well-known numerical benchmark. A sphere moves with a velocity $U$ along the centre line of a cylinder with a diameter twice as large as the sphere. The cylinder extends to infinity and the flow rate in each cross section is zero ${ }^{1}$. In our simulation the sphere is instantaneously accelerated to a steady velocity $U$ moving through a fluid which was initially at rest. Due to the visco-elastic nature of the fluid the drag force on the cylinder gradually builds up until a steady state value is attained. This steady state value will be compared against the results reported by other groups.

Instead of letting the sphere move we consider the same problem in a frame of reference that moves with the sphere. In this frame the sphere is at rest, the cylinder wall moves with the velocity $-U$ and the flow rate in each cross section is $-\pi R^{2} U$. Since we neglect inertia, the equations in the moving frame are identical to the 'laboratory' frame of reference.

In the simulations we use a flow domain which is 30 sphere radii long. The ends of the tube are connected by periodic boundary conditions. We assume no-slip boundary conditions on the cylinder wall and prescribe the flow rate in the tube. The basic mesh is depicted in Fig. 4. In the flow computations we use uniform refinement of this basic mesh M1. The coarsest mesh used in the computations, M2, consists of 272 elements whereas the finest mesh used, M5, contains 1700 elements.

We will make the problem dimensionless using the sphere radius $R$ to scale the spatial dimensions and the relaxation time $\lambda$ as the characteristic time scale used. The Weissenberg number is therefore given by

$$
\mathrm{We}=\frac{\lambda U}{R},
$$

The drag force is divided by the Stokes drag on a sphere moving with the same velocity in an infinite expanse of fluid. The dimensionless drag force thus becomes

$$
K=\frac{F}{6 \pi \eta R U}
$$

\footnotetext{
${ }^{1}$ This requirement tries to match an experimental setup of a cylinder with a bottom.
} 


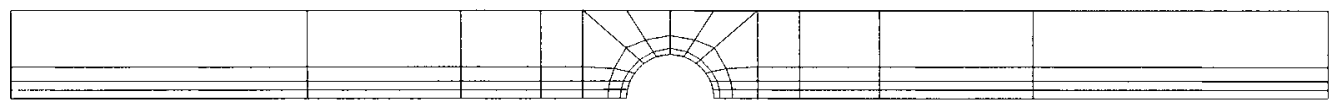

Fig. 4. The primary mesh M1 having 68 elements. The meshes used are uniform refinements of this mesh. The coarsest mesh used in the calculations, M2, is two times refined in both directions, M3 is three times refined etc. The finest mesh used is M5. The domain is 30 sphere radii long. Inlet and outlet are connected by means of periodic boundary conditions.

For the finest mesh (M5, 1700 elements) with the smallest time step $\left(\Delta t=5 \times 10^{-4}\right.$ in dimensionless units), a simulation of 24000 time steps using 100 fields took about $24 \mathrm{~h}$ on a single processor of a HP9000-J282 workstation. For this time step we used a discretisation of the memory function using the following refinement (see Fig. 3) of $\{5 \times \Delta t, 5 \times 2 \Delta t, 7 \times 4 \Delta t, 9 \times 8 \Delta t, 10 \times 16 \Delta t, 10 \times$ $32 \Delta t, 10 \times 64 \Delta t, 10 \times 128 \Delta t, 10 \times 256 \Delta t, 10 \times 512 \Delta t, 14 \times 1028 \Delta t\}$. Adding everything up this yields a large cut-off time of 12.27 relaxation times. This is the cut-off time taken in most of the calculations.

We typically need a much smaller time step for the evolution of the fields than for the calculation of the stress integral. The reason is that in the present code a simple first-order Euler-forward scheme is used for the time evolution of the deformation fields. This requires small time steps in order to obtain accurate results. The time step required for stability (CFL condition) is much larger. For the stress integral a second-order scheme is used, and larger time steps are allowed here. The first few intervals of the age discretisation are used to bridge the time interval needed for updating the deformation fields and that for doing the stress integral. If, for example, we use a twice as large time step as used above (thus $\left.\Delta t=10^{-3}\right)$ we join the first two intervals $\left(5 \times 5 \times 10^{-4}, 5 \times 1 \times 10^{-3}\right)$ and change that to 10 steps of $1 \times 10^{-3}$. Now the new discretisation, again using 100 fields, becomes $\{10 \times \Delta t, 7 \times 2 \Delta t$, $9 \times 4 \Delta t, 10 \times 8 \Delta t, 10 \times 16 \Delta t, 10 \times 32 \Delta t, 10 \times 64 \Delta t, 10 \times 128 \Delta t, 10 \times 256 \Delta t, 14 \times 512 \Delta t\}$. So after the bridging of the difference in time steps the whole discretisation stays the same, except for a small change in the first intervals. Having experimented with several other discretisations, both using more and less fields, we concluded that the error introduced by the discretisation presented above using 100 fields is much smaller than the errors caused by the time evolution of the deformation fields. Optimisation of the truncation error may lead to a number of fields that is smaller than 100, but we have not explored this possibility yet.

The time evolution of the drag for mesh M5 is plotted in Fig. 5 for various values of We. To obtain the stationary values, the drag was monitored up to 12 times the relaxation time. For such large times the asymptotic values of the drag are very closely approached. To determine the asymptotic value we fitted the tail $(t>8 \lambda)$ to a constant plus a decaying exponential. As will be discussed below, for high Weissenberg number the calculation becomes unstable before $t=12 \lambda$ can be reached. For these Weissenberg numbers we did the extrapolation for a time interval that started earlier, giving slightly less accurate results. For higher Weissenberg numbers (we only tried We $=2.0$ ) the computations become unstable very quickly and no useful results can be obtained.

In the current implementation, the evolution of the deformation fields is the most important part affecting accuracy and stability. The first graph in Fig. 6 shows the relative difference of the computed drag compared with values reported in literature for different meshes at various Weissenberg numbers. The literature values are taken from Warichet and Legat [10], which are considered to be the most accurate up to now. The time step used is $1 \times 10^{-3}$. The results for the meshes M4 an M5 superimpose, but the error does not vanish. This is due to the fact that even in the stationary case an error due to the 


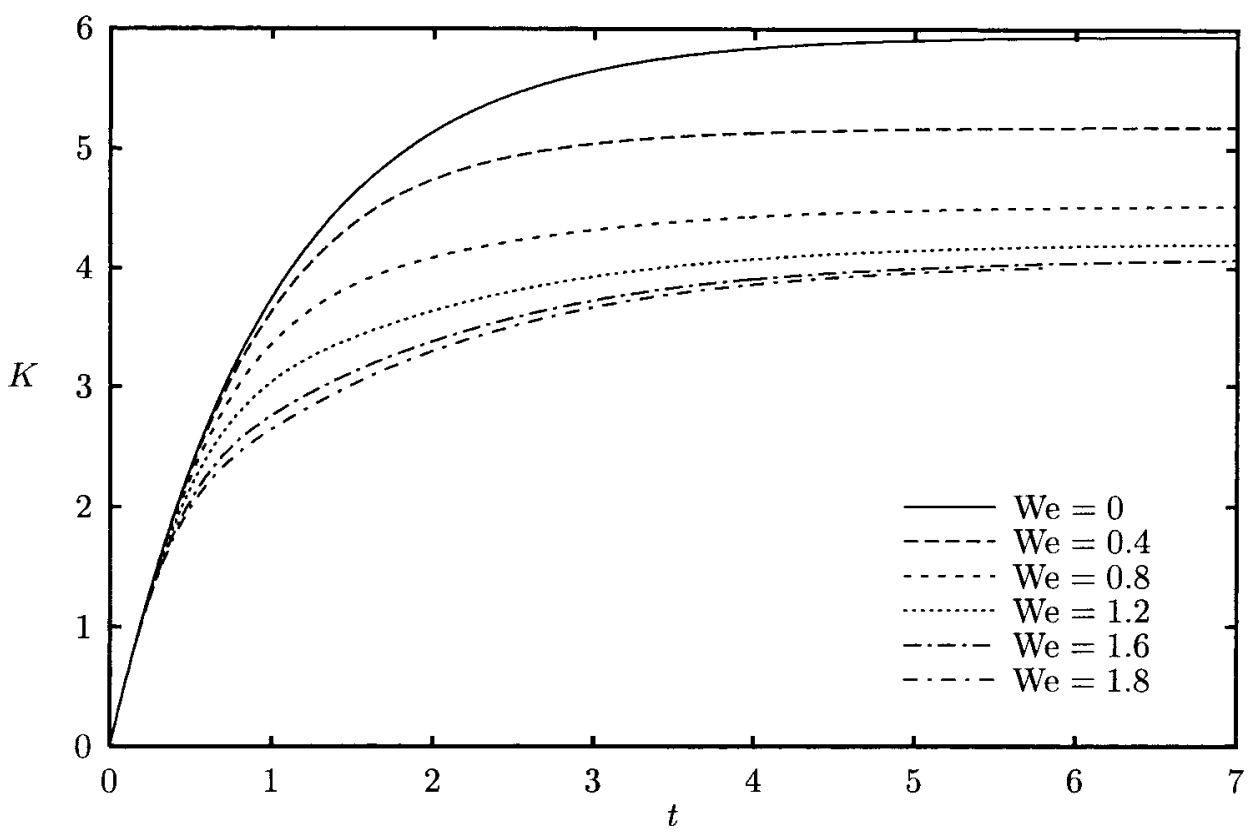

Fig. 5. The time evolution of the dimensionless drag $K$ for the $1: 2$ geometry.

time discretisation persists. The solution of the evolution equations for the deformation fields Eq. (5) does not become stationary. Even if the velocity field $\boldsymbol{v}(\boldsymbol{x}, t)$ is stationary, a Finger tensor field created at some reference time $t^{\prime}$ will keep being deformed. This gives rise to a permanent time discretisation error in the (B) $t_{t}$ 's and thus in the stress, which is a weighted sum of these tensors.

The second graph of Fig. 6 shows the time step convergence of the dimensionless drag using the most refined mesh M5. Three time steps were studied $5 \times 10^{-3}, 1 \times 10^{-3}$ and $5 \times 10^{-4}$. As can be seen very small time steps have to be used to obtain high accuracy results. This is basically a result of the first-order time discretisation being used for the evolution equation of the deformation fields. A small time step is inconvenient because the number of time steps needed increases and thus also the total CPU time. This situation can be improved by using higher-order time integration, but we have not done that yet.

A more serious problem is the occurrence of instabilities, which are related to the oldest deformation fields becoming too distorted. Because in the evolution of the deformation fields there is no relaxation mechanism, errors are not damped. When fields become too distorted, errors grow too rapidly and the field computation may become unstable. If the error in the oldest fields become too large it significantly contributes to the stress and causes the full problem to become unstable.

In Fig. 7 we monitored the first occurrence of instability ${ }^{2}$ in the drag for the startup problem we are considering. This time of onset of instability is indicated by $t_{\text {inst }}$. In order to avoid any influence of the

\footnotetext{
${ }^{2}$ The first occurrence of the instability is defined as the point where there is a significant visible 'jump' in the drag, which seems to be rather independent from the time discretisation. After this jump the flow becomes unstable.
} 



Fig. 6. Mesh convergence and time convergence for the UCM case of the dimensionless drag force $K$ relative to the most accurate values reported in the literature [10]. The first graph demonstrates mesh convergence for different uniformly refined meshes. The time step used is $1 \times 10^{-3}$. The second graph shows the linear convergence toward for decreasing time steps. Here mesh M5 is used. 



Fig. 7. The time of the onset of instability $t_{\text {inst }}$ in the simulations is monitored as function of for mesh M3 (top figure) and mesh refinement for $\mathrm{We}=1$ and $\mathrm{We}=1.7$ (bottom figure).

cut-off time here, the value of $\tau_{\mathrm{c}}$ is taken to be considerably larger than $t_{\text {inst }}$. The dependence of $t_{\text {inst }}$ on the Weissenberg number for mesh M3 is depicted in the first graph of Fig. 7. It appears that in the range $\mathrm{We}=1.4-1.6$ the type of instability changes. For the first 'branch' we find that the time of instability 
can approximately be described by a linear curve given by

$$
\frac{U t_{\text {inst }}}{R}=11.7+4 \mathrm{We}
$$

In terms of the time scale $\lambda$ this can also be written as

$$
\frac{t_{\text {inst }}}{\lambda}=\frac{11.7}{\mathrm{We}}+4
$$

which shows that $t_{\text {inst }}$ becomes smaller with respect to the relaxation time $\lambda$ for larger values of We. No instabilities are found below We $=0.8$ for mesh M3. This indicates that for small Weissenberg numbers the growth rate of the errors in the fields is smaller than the rate of decay of the memory function in the stress calculations. The second graph in Fig. 7 depicts the mesh dependency of $t_{\text {inst }}$ for We $=1$ and $\mathrm{We}=1.7$. For a more refined mesh the instability occurs later for $\mathrm{We}=1$, but for $\mathrm{We}=1.7$ the result is less regular and for mesh M5 the time of instability decreases compared to mesh M4. Further refinement of the mesh is needed to see whether we find real mesh convergence for the higher Weissenberg numbers. However, this will require more computer resources and will be part of future work. The dependence on the time step is also shown in Fig. 7 for We $=1$. As can be seen there is almost no time step dependence.

A simple remedy for the occurrence of instabilities that comes to mind is reducing the cut-off time $\tau_{\mathrm{c}}$. In this way the 'life time' of a field is reduced such that it cannot become distorted too much and generate an instability. We have done various computations that confirm this statement. Furthermore, it seems that the $t_{\text {inst }}$ as shown in Fig. 7 is a good measure for the maximum cut-off time $\tau_{\mathrm{c}}$ that removes the instability. At first, this is somewhat surprising since $t_{\text {inst }}$ is measured in the start-up flow. However, note that when starting to move the sphere one immediately enforces a Stokesian flow field having a flow rate that does not change during the computations. Depending on the Weissenberg number the stationary flow field will differ from the initial field, but not significantly. Therefore, the deformation fields experience an approximately stationary flow field even in the start-up flow. The practical solution is thus to make the cut-off time small enough. A small cut-off time, however, introduces an inaccuracy in the stress integral. We found that imposing a small enough cut-off time does not give significant inaccuracies for smaller than or equal to 1.4. For higher Weissenberg numbers the time of instability is smaller, as can be seen in Fig. 7. This means that the cut-off times that have to be taken to avoid the instability dominate the errors in the drag and a comparison with literature values would not be meaningful.

The stationary values of the dimensionless drag $K$ are plotted in Fig. 8 and listed in Table 1 as a function of We. The results are obtained by using the most refined mesh (M5) and by extrapolating the results of the drag for different time steps to $\Delta t=0$ (see Fig. 6). Furthermore, we used a cut-off time $\tau_{\mathrm{c}}=12.27 \lambda$ for all calculations. This means, that for the higher Weissenberg numbers the cut-off time is too small to avoid instabilities and stationary flow cannot be maintained to very large times. As explained earlier, this is not a problem here since we use extrapolation to obtain the stationary drag.

Currently the maximum (reliable) Weissenberg number, computed by the most sophisticated methods, is $2.6([10,11])$. The methods used to reach these high Weissenberg numbers are time independent methods. With our time dependent method we reach We $=1.8$. Beyond this value the flow becomes unstable too quickly to obtain reliable stationary drag values. The values for the drag on the 


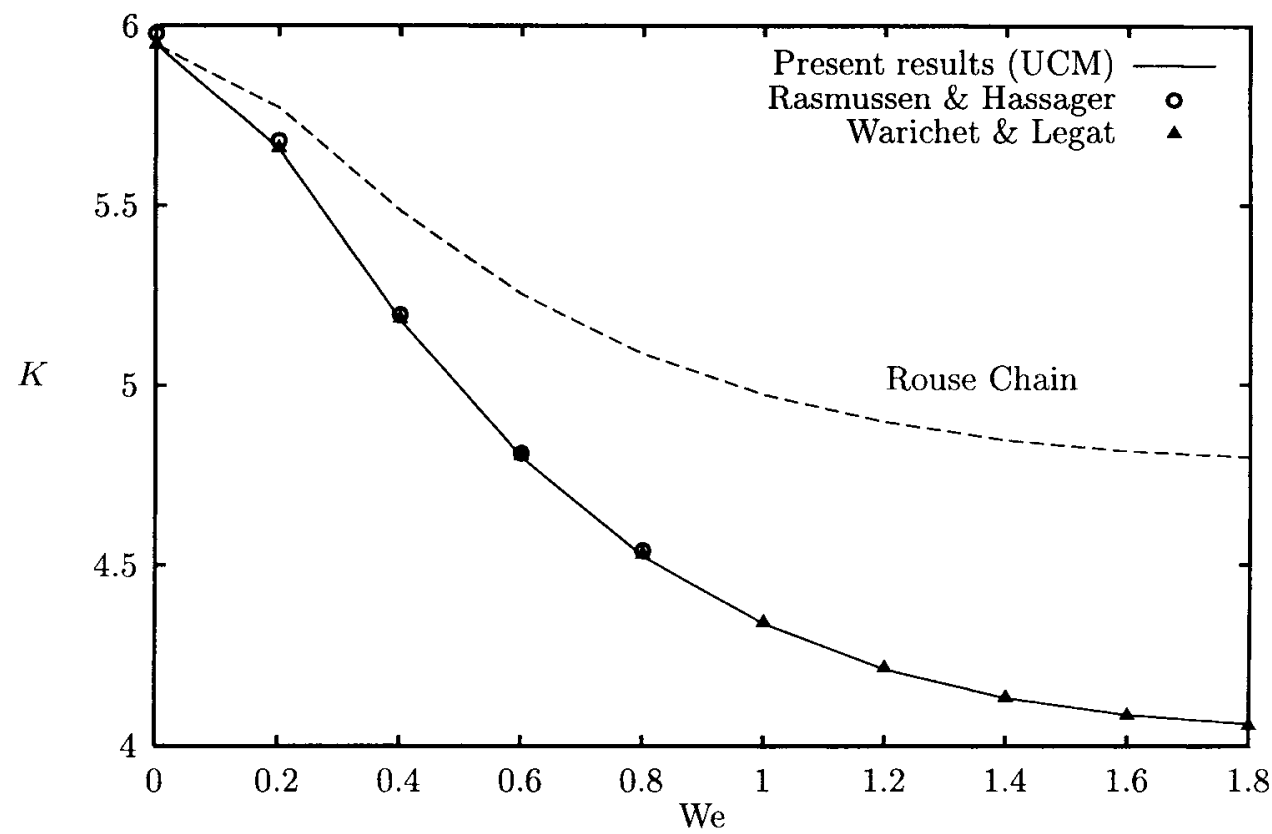

Fig. 8. The dimensionless drag $K$ versus the Weissenberg number We, for the $1: 2$ geometry.

Table 1

Drag coefficient $K$ for the $1: 2$ geometry $^{\text {a }}$

\begin{tabular}{lllll}
\hline We & $\begin{array}{l}\text { Present } \\
\text { results }\end{array}$ & $\begin{array}{l}\text { Warichet and } \\
\text { Legat [10] }\end{array}$ & $\begin{array}{l}\text { Baaijens } \\
\text { et al. [11] }\end{array}$ & $\begin{array}{l}\text { Rasmussen and } \\
\text { Hassager [5] }\end{array}$ \\
\hline 0 & 5.9475 & 5.9469 & 5.947 & 5.979 \\
0.2 & 5.6585 & 5.6592 & 5.660 & 5.679 \\
0.4 & 5.1846 & 5.1862 & 5.186 & 5.198 \\
0.6 & 4.7987 & 4.8009 & 4.801 & 4.809 \\
0.8 & 4.5249 & 4.5299 & 4.528 & 4.54 \\
1.0 & 4.3381 & 4.3405 & 4.341 & 4.216 \\
1.2 & 4.2131 & 4.2159 & 4.134 & \\
1.4 & 4.1327 & 4.1336 & 4.084 & \\
1.6 & 4.0856 & 4.0831 & 4.057 & \\
1.8 & 4.0603 & 4.0557 & 2.6 & 0.8 \\
Maximum & 1.8 & 2.6 & & \\
\hline
\end{tabular}

${ }^{a}$ Present results are compared with recent results from literature. The results from Rasmussen and Hassager [5] were obtained by a Lagrangian technique for solving Rivlin-Sawyers equations time dependently. The results are plotted in Fig. 8 .

sphere in literature, computed by different methods, agree up to the third significant digit. The numbers for the stationary drag coefficient are compared in Table 1. Compared to the values of Warichet and Legat [10] and Baaijens et al. [11] our values differ only by $0.01 \%$ for low up to $0.1 \%$ for high We. 


\subsection{The Rouse chain}

One of the strong points of the deformation field method is its flexibility to handle any memory function. The memory function, which can even be determined experimentally using linear viscoelastic data, is only used to determine the weights of the different fields. So, an extension to more complex memory functions does not generate additional computational costs. This in contrast to, e.g. a differential constitutive equation which would require a multi-mode analysis leading to significantly longer computer runs. To demonstrate that very many modes can be handled without a problem, a Rouse chain of 100 modes is simulated. The memory function in this case becomes

$$
M(\tau)=\sum_{k=1}^{100}\left(\frac{\sin [\pi k / 202]}{\sin [\pi / 202]}\right)^{2} \exp \left[-\left(\frac{\sin [\pi k / 202]}{\sin [\pi / 202]}\right)^{2} \frac{\tau}{\lambda}\right]
$$

Results using mesh M4 and $\Delta t=5 \times 10^{-4}$ are plotted in Fig. 8. In this calculation the Weissenberg number is based upon the largest relaxation time in the spectrum, i.e. $\lambda$.

\subsection{The Papanastasiou-Scriven-Macosko model}

To our knowledge the only time-dependent implementation of a non-trivial Rivlin-Sawyers model, that appeared in the literature so far is by Rasmussen and Hassager [1]. The model they use is the Papanastasiou-Scriven-Macosko (PSM) model, belonging to the class of Rivlin-Sawyers equations. The constitutive equation for the PSM model is given by

$$
\tau=\frac{\eta_{\mathrm{p}}}{\lambda^{2}} \int_{-\infty}^{t} \frac{\alpha}{\alpha-3+\beta I_{1}+(1-\beta) I_{2}} \exp \left[\frac{\left(t^{\prime}-t\right)}{\lambda}\right] \boldsymbol{B}_{t^{\prime}} \mathrm{d} t^{\prime}
$$

where $\eta_{\mathrm{p}}$ is the contribution of the extra stress $\tau$ to zero-shear-rate viscosity and $I_{1}$, and $I_{2}$ are the first and second invariants of $\boldsymbol{B}_{t}^{\prime}$. This model has the convenient property that one parameter $(\beta)$ has no influence on the behaviour of the model in simple shear flow. This parameter can thus be used to fit the elongational behaviour without affecting the properties in shear flow. For high values of the parameter the model effectively becomes identical to the UCM model.

Rasmussen and Hassager [1] compared their computational results to experimental data obtained by Becker et al. [12] on spheres with a radius of $1.27 \mathrm{~cm}$ settling in a cylindrical tube with a radius of $5.23 \mathrm{~cm}$. The fluid is characterised in shear flow which resulted in the following values of the parameters: $\eta_{\mathrm{s}}=12.37 \mathrm{~Pa} \mathrm{~s}, \quad \eta_{\mathrm{p}}=8.60 \mathrm{~Pa} \mathrm{~s}, \lambda=1.21 \mathrm{~s}$ and $\alpha=25000$. Note that a viscous contribution (solvent) is present in the model. We have neglected inertia in our calculations. The effect of inertia in this problem (the elasticity number is equal to $38.1[12,1]$ ) is very small. We did some calculations with inertia included and the drag difference was less than $0.1 \%$ for the Weissenberg numbers considered here. The parameter $\beta$ was varied in the simulation to explore the effect of the elongational properties on the settling velocity. The value of $\alpha$ is very large so only for large deformations, i.e. large, deviations from the UCM fluid will occur. The largest deformations in the falling sphere geometry occur near the centreline, in the wake of the cylinder, where strong elongational flow is present. 


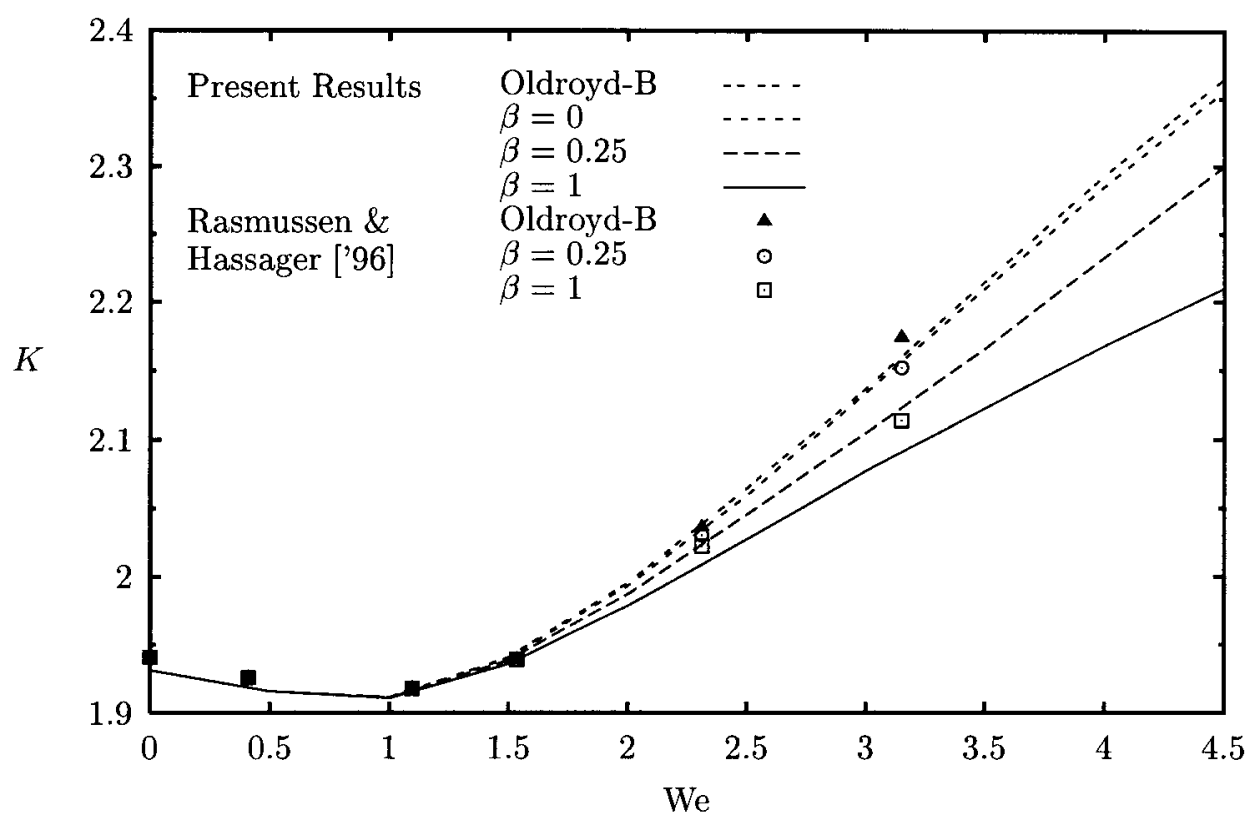

Fig. 9. The drag coefficient $K$ as a function of for the PSM model. Values are compared to data of Rasmussen and Hassager [1].

The mesh being used is similar to that of mesh M4 in the UCM computation, however with an extra rectangular region between the previous position of the wall (two times the sphere radius) and the new position of the wall. The time step used is $1 \times 10^{-3}$. The stationary results are shown in Fig. 9 and are obtained in the same way as for the UCM model (see Section 5.1). The results of Rasmussen and Hassager [1] show the same trends as our results. For the We $\rightarrow 0$ limit we obtain the exact Newtonian result $K=1.931$ [13]. In the regime where the results for different $\beta$ 's superimpose our results superimpose with those of [1] (see Fig. 9). For higher values of the number models with a different value of $\beta$ start to generate different predictions for the drag force. In this regime the deformations in the flow are apparently large enough to make a difference between the models with different values of $\beta$. It is also clear that there is a small (less than 1.5\%), but significant difference between our results and those obtained by Rasmussen and Hassager.

\section{Conclusions}

A time-dependent Eulerian method to use integral constitutive equations for the flow through complex geometries was developed. We implemented the method for time-separable Rivlin-Sawyers equations. To do this we introduced deformation fields. The method is relatively efficient, since it only uses 100 tensor fields. This is small compared to e.g. stochastic configuration field methods that use 1000 vector fields as a minimum [3]. However, it is less efficient than differential models if only a small number of relaxation times are used.

The integral over the deformation history is approximated by a finite sum over all fields, which is second order in time. No particle tracking is used, only the field values at the current position and time 
are needed to compute the stress. The deformation fields are convected and deformed by the flow. The evolution equation of the fields is solved by a standard finite element technique (DG) and a first-order explicit scheme in time. The latter scheme is the weakest point in our current implementation. Since even for stationary situations an error due to the time discretisation persists, very small time steps were needed. Using a higher-order scheme would be a major improvement and will be part of our future work.

The deformation fields remain unsteady and old fields can become too distorted and generate instabilities. These instabilities can be avoided by limiting the cut-off time in such a way that old fields are removed before becoming too distorted. The additional error is dependent on the mesh and the Weissenberg number but is usually small. However, for high Weissenberg numbers accuracy may be affected and it is important to check the magnitude of the error.

The accuracy of the method was investigated by comparing our simulation of the moving sphere UCM benchmark with the most accurate values available from literature. We have shown that our method converges to the literature values when refining the spatial and the time discretisation. The possibility to use arbitrary spectra has been demonstrated by simulations of a Rouse model with 100 modes. The possibility to incorporate nonlinear effects has been illustrated using a PSM model.

In literature two different approaches to solve integral constitutive equations are known. The first is an Eulerian method based on streamline integration and is restricted to steady flows. The second method is a Lagrangian method, where the mesh deforms with the flow and therefore requires frequent remeshing to avoid highly distorted elements. The deformation field method combines the advantage of an Eulerian method (no remeshing) with the distinct feature of a Lagrangian method (timedependence).

Since the deformation fields store the full history of deformation the constitutive models that can be handled are not restricted to single integral models. In fact, any model that obeys the usual assumptions of simple fluid theory (locality, fading memory) can, in principle, be implemented using deformation fields. This opens up the possibility to explore all kinds of new models that are currently being developed, such as the models based on reptation and convective constraint release.

\section{Appendix A}

\section{A Reduction of the number of Finger tensor components}

Convecting and deforming deformation fields is relatively expensive. Therefore, any reduction in the number of components that need to be computed reduces the storage and CPU requirement proportionally. In an incompressible flow the determinant of the Finger tensor is equal to 1 . This means that the tensor components are dependent. Using this dependency the number of tensor components can be reduced by one and thus eliminating the need to store this component in a field.

The Finger tensor has also the property that it is positive definite. The continuous evolution equations make sure that this positive definiteness is maintained. This is not necessarily the case for the discretised evolution equations. Numerical errors can cause the positive definiteness to be violated. We devised a transformation that simultaneously explicitly imposes the $\operatorname{det}(\boldsymbol{B})=1$ and the positive definiteness. We will illustrate this procedure for the axisymmetric case. The planar case is more simple (take $\left.B_{\theta \theta}=1, r \rightarrow \infty\right)$. 
In cylindrical coordinates the Finger tensor is given by

$$
\left(\begin{array}{llc}
B_{r r} & 0 & B_{r z} \\
0 & B_{\theta \theta} & 0 \\
B_{r z} & 0 & B_{z z}
\end{array}\right), \quad \text { with } \quad\left(B_{r r} B_{z z}-B_{r z}^{2}\right) B_{\theta \theta}=1
$$

The requirement of positive definiteness in this case gives rise to four constraints on the components:

$$
B_{r r}>0, \quad B_{z z}>0, \quad B_{r r} B_{z z}-B_{r z}^{2}>0, \quad B_{\theta \theta}>0
$$

The following linear transformation will be applied

$$
\mu=\sqrt{B_{\theta \theta}}\left[\frac{B_{r r}+B_{z z}}{2}\right], \quad \nu=\sqrt{B_{\theta \theta}}\left[\frac{B_{r r}-B_{z z}}{2}\right], \quad \xi=\sqrt{B_{\theta \theta}} B_{r z} .
$$

The constraint $\operatorname{det}(\boldsymbol{B})=1$ gives

$$
\mu=\sqrt{1+\nu^{2}+\xi^{2}}
$$

The positive root has to be applied because of positive definiteness, which gives $\mu>0$.

If one numerically can make sure that $\sqrt{B_{\theta \theta}}$ is positive the positive definiteness of the whole tensor is guaranteed. This becomes clear by performing the inverse transformation

$$
\begin{aligned}
& B_{r r}=\frac{\mu+\nu}{\sqrt{B_{\theta \theta}}}=\frac{\sqrt{1+\nu^{2}+\xi^{2}}+\nu}{\sqrt{B_{\theta \theta}}}, \\
& B_{z z}=\frac{\mu-\nu}{\sqrt{B_{\theta \theta}}}=\frac{\sqrt{1+\nu^{2}+\xi^{2}}-\nu}{\sqrt{B_{\theta \theta}}}, \\
& B_{r r} B_{z z}-B_{r z}^{2}=\frac{1}{B_{\theta \theta}} .
\end{aligned}
$$

The evolution equation for $\nu, \xi, \mu$ and $\sqrt{B_{\theta \theta}}$ are

$$
\begin{aligned}
& \frac{\mathrm{D} \nu}{\mathrm{D} t}=\left[\frac{\partial v_{r}}{\partial r}-\frac{\partial v_{z}}{\partial z}\right] \mu+\left[\frac{\partial v_{r}}{\partial z}-\frac{\partial v_{z}}{\partial r}\right] \xi \\
& \frac{\mathrm{D} \xi}{\mathrm{D} t}=\left[\frac{\partial v_{r}}{\partial z}+\frac{\partial v_{z}}{\partial r}\right] \mu+\left[\frac{\partial v_{z}}{\partial r}-\frac{\partial v_{r}}{\partial z}\right] \nu, \\
& \mu=\sqrt{1+\nu^{2}+\xi^{2}} \\
& \frac{\mathrm{D}}{\mathrm{D} t} \frac{\sqrt{B_{\theta \theta}}}{r}=0 .
\end{aligned}
$$

Notice that $\nu$ and $\xi$ evolve completely independent of $B_{\theta \theta}$. 


\section{References}

[1] H.K. Rasmussen, O. Hassager, On the sedimentation velocity of spheres in a polymeric liquid, Chem. Eng. Sci. 51 (1996) 1431-1440.

[2] M. Laso, H.C. Öttinger, Calculation of viscoelastic flow using molecular models: the CONNFFESSIT approach, J. NonNewtonian Fluid Mech. 47 (1993) 1.

[3] M.A. Hulsen, A.P.G. van Heel, B.H.A.A. van den Brule, Simulation of viscoelastic flows using Brownian configuration fields, J. Non-Newtonian Fluid Mech. 70 (1997) 79-101.

[4] X. Gallez, P. Halin, G. Lielens, R. Keunings, V. Legat. The adaptive Lagrangian particle method for macroscopic and micro-macro computations of time-dependent viscoelastic flows, Comput. Meth. Appl. Mech. Eng., 1998, submitted for publication.

[5] H.K. Rasmussen, O. Hassager, Simulation of transient viscoelastic flow, J. Non-Newtonian Fluid Mech. 46 (1993) 289305.

[6] H.K. Rasmussen, O. Hassager, Simulation of transient viscoelastic flow with second-order time integration, J. NonNewtonian Fluid Mech. 56 (1995) 65-84.

[7] A. Goublomme, B. Draily, M.J. Crochet, Numerical prediction of extrudate swell of a high-density polyethylene, J. NonNewtonian Fluid Mech. 44 (1992) 171-195.

[8] D. Rajagopalan, R.C. Armstrong, R.A. Brown, Comparison of computational efficiency of flow simulations with multimode constitutive equations: integral and differential models, J. Non-Newtonian Fluid Mech. 46 (1993) $243-273$.

[9] R. Guénette, M. Fortin, A new mixed finite element method for computing viscoelastic flows, J. Non-Newtonian Fluid Mech. 60 (1995) 27-52.

[10] V. Warichet, V. Legat, Adaptive high-order prediction of the drag correction factor for the upper-convected Maxwell fluid, J. Non-Newtonian Fluid Mech. 73 (1997) 95-114.

[11] F.P.T. Baaijens, S.H.A. Selen, H.P.W. Baaijens, G.W.M. Peters, H.E.H. Meijer, Viscoelastic flow past a confined cylinder of a low density polyethylene melt, J. Non-Newtonian Fluid Mech. 68 (1997) 173-203.

[12] L.E. Becker, G.H. McKinley, H.K. Rasmussen, O. Hassager, The unsteady motion of a sphere in a viscoelastic fluid, J. Rheol. 38 (1994) 377-403.

[13] J. Happel, J. Brenner. Low Reynolds Number Hydrodynamics Martinus, Nijhoff, Dordrecht, 1983. 